\title{
Analytical Calculation of Volumes-of-Intersection for Iterative, Fully 3-D PET Reconstruction
}

\author{
Jürgen J. Scheins*, Fritz Boschen, Member, IEEE, and Hans Herzog, Member, IEEE
}

\begin{abstract}
Use of iterative algorithms to reconstruct three-dimensional (3-D) positron emission tomography (PET) data requires the computation of the system probability matrix. The pure geometrical contribution can easily be approximated by the length-of-intersection (LOI) between lines-of-response (LOR) and individual voxels. However, more accurate geometrical projectors are desirable. Therefore, we have developed a fast method for the analytical calculation of the 3-D shape and volume of volumesof-intersection (VOI). This method provides an alternative robust projector with a uniformly continuous sampling of the image space. The enhanced calculation effort is facilitated by using several speedup techniques. Exploiting intrinsic symmetry relations and the sparseness of the system matrix allows to create an efficiently compressed matrix which can be precomputed and completely stored in memory. In addition, a new voxel addressing scheme has been implemented. This scheme avoids time-consuming symmetry transformations of voxel addresses by using an octant-wise symmetrically ordered field of voxels. The above methods have been applied for a fully 3-D, iterative reconstruction of 3-D sinograms recorded with a Siemens/CTI ECAT HR+ PET scanner. A comparison of the performance of the reconstruction using LOI weighting and VOI weighting is presented.
\end{abstract}

Index Terms - Iterative reconstruction, memory-resident system matrix, positron emission tomography (PET), symmetries, volumes-of-intersection (VOI).

\section{INTRODUCTION}

I TERATIVE fully three-dimensional (3-D) positron emission tomography (PET) reconstruction remains a challenging computational task due to the tremendous number of measured lines-of-response (LOR) for multiring PET systems in 3-D mode. The improved spatial information and gain of sensitivity of 3-D measurements needs the incorporation of all detected LORs to obtain optimal image quality [1]. Especially, in the context of recently available low-cost computation power list-mode (LM) reconstruction is gaining growing importance, e.g., [2] and [3]. However, the calculation and management of the extremely large system matrix requires sophisticated techniques to achieve acceptable performance. Data compression due to rebinning [4] and simplified detection models still remain a good compromise between effort and image quality. Usually applications follow a simple modeling of the detection probabilities due to the prohibitive problem of time-intensive calculations and

Manuscript received March 31, 2006; revised June 8, 2006. This work was supported by the German Research Foundation under Grant HE 3090/1. Asterisk indicates corresponding author.

*J. J. Scheins is with the Institute of Medicine, Research Center Jülich, Jülich, 52425 Germany (e-mail: j.scheins@fz-juelich.de).

F. Boschen is with the Institute of Communication Theory, University of Wuppertal, Wuppertal D-42097 Germany.

H. Herzog is with the Institute of Medicine, Research Center Jülich, Jülich, 52425 Germany.

Digital Object Identifier 10.1109/TMI.2006.880679 missing possibility of storing matrix weights. For simplified models, approximate weights can be calculated on-the-fly, e.g., the length-of-intersection (LOI) between detected LORs and individual voxels [5]. In this case the implementation proves to be rather straightforward and the calculation time remains acceptable. In contrast, a more precise modeling without significantly increasing the reconstruction time is only feasible with other strategies. Our current approach addresses this issue, utilizing a more appropriate volume-based geometrical projector in conjunction with a memory-resident system matrix.

The applied geometrical projector essentially influences the quality of the reconstructed images. For the two-dimensional (2-D) case, the transition from LOI weighting to area-of-intersection weighting provides an improved measure of the geometrical detection probability when the macroscopic crystal sizes should be taken into account [6]-[8]. Analogously, for the 3-D case polyhedrons representing specific voxel fragments of covered tubes-of-response (TOR) have to be considered. Therefore, as 3-D geometrical projector, we have developed a new method for the analytical calculation of the shape and volume of occurring volumes-of-intersection (VOI). Thus, the system matrix can be built, providing a uniformly continuous sampling of the image space. In addition, our implementation includes several speedup techniques to get reconstruction times adequate for routine applications without need of computational parallelization. The precomputation of weights, the full storage in RAM as well as a new symmetry handling scheme are key elements to reduce the computing time.

The new analytical VOI projector and the accelerated symmetry handling have been integrated into a modular framework which currently facilitates a fully 3-D reconstruction of 3-D sinograms acquired by a Siemens/CTI HR+ scanner. As iterative methods the maximum likelihood expectation maximization (MLEM) and ordered subset expectation maximization (OSEM) algorithm [9]-[11] have been used so far. However, our approach is not restricted to these algorithms. Also more advantageous methods including more appropriate data correction models, e.g., weighted OSEM [12], Poisson models [13]-[17], or maximum a posteriori reconstruction [18], [19], are applicable. In this context, we present preliminary results based on precorrected and rebinned 3-D sinogram data that cannot be regarded as optimized reconstruction method, but allow to validate objectively our methods.

\section{Materials AND MethodS}

\section{A. Analytical Calculation of VOI}

1) Computational Representation of Polyhedrons: For a volume-based projector, the fundamental objects are poly- 
hedrons, so that the analytical calculations can be reduced to qualified operations, referring exclusively to polyhedrons. Each polyhedron can be characterized by a specific number of vertices $(>3)$ in the 3-D space. To uniquely define its 3-D structure, the limiting surfaces have to be known as well. Each surface is represented by a closed polygon connecting various vertices in a plane with a specific orientation in the 3-D space. Consequently, one list of vertices and one list of polygons constitute the building blocks of any polyhedron. These building blocks have been integrated in one $\mathrm{C}++$ class. Each surface polygon is organized as a chained list of subsequent vertices, and adjacent polygons can share various vertices. For example, a simple cuboid consists of eight vertices and six surface polygons, i.e., rectangles, each connecting a combination of four vertices. Technically, the constituting vertex coordinates of each polygon are explicitly implemented as references to the corresponding data members in the list of unique vertices. Thus linear coordinate transformations must be applied only to the members of the list of vertices to execute a fast transformation of the polyhedron as a whole.

2) Determination of VOI Elements: The determination of the shape of involved VOI can be reduced to the task of splitting polyhedrons at any user-defined plane in the 3-D space. The TOR geometry can be translated into a specific polyhedron and the relevant separation planes correspond to the surfaces of the voxels. Then subsequent splitting of the polyhedron at all "voxel planes" provides all voxel fragments as independent subpolyhedrons. Each subpolyhedron is assigned to exactly one unique image voxel and represents a specific fraction of its volume which is "observed" by the generator TOR.

Having understood any TOR as a unique polyhedron in 3-D space, the determination of separated voxel fragments can easily be performed. Any separation plane splits a polyhedron into two unique subpolyhedrons. Only the intersection points between the separating plane and the lines which directly connect vertices on opposite sides of this plane have to be calculated. All intersection points form an additional surface, i.e., an intersection polygon located in the separation plane and shared in both subpolyhedrons. Finally, all constituting components together have to be redistributed into independent building blocks forming the two separated polyhedrons.

Note, the same algorithm of splitting at "voxel planes" can also be applied for the simple LOI weighting. Instead of splitting, a complex polyhedron a single LOR is now used. As LOR the central line of the corresponding TOR is taken and the 3 -D splitting provides the voxel associated line elements whose lengths fix the LOI weights.

3) Analytical Volume Calculation of VOI Elements: The analytical calculation of the volume $V$ of a complex polyhedron $\mathcal{P}$ can be realized through the reformulation of the volume integral

$$
V=\iiint_{\mathcal{P}} d x d y d z=\int_{\mathcal{P}_{z}} A(z) d z
$$

with

$$
A(z)=\iint_{\mathcal{P}^{\prime}(z)} d x d y .
$$

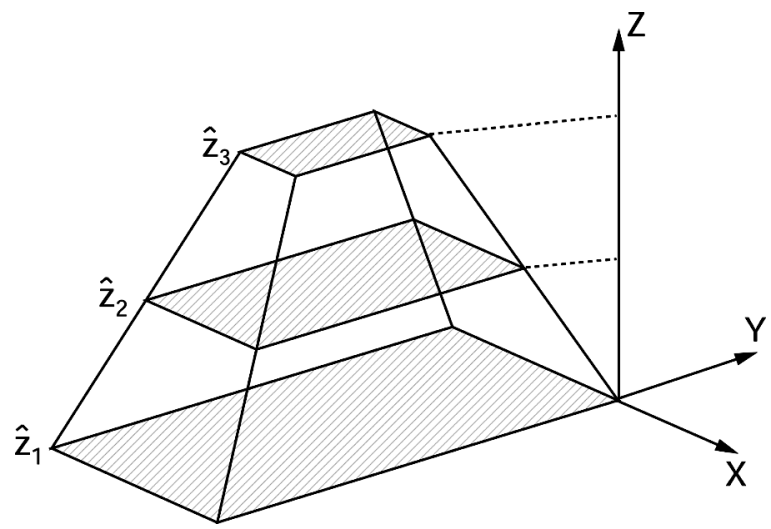

Fig. 1. Evaluation of three cross sections in the $X Y$ plane at different values of the $z$ coordinate $\left(\hat{z}_{1}, \hat{z}_{2}, \hat{z}_{3}\right)$ for a given simple polyhedron. Cross sections $\left(A\left(\hat{z}_{1}\right)=A_{1}, A\left(\hat{z}_{2}\right)=A_{2}, A\left(\hat{z}_{3}\right)=A_{3}\right)$ can be calculated by decomposing each sampled polygon into elementary triangles.

The function $A(z)$ gives the cross section of the polyhedron for each value of the coordinate along the $z$ axis $^{1}$ and the volume of the polyhedron is given by the corresponding integral of $A(z)$ within the appropriate bounds (Fig. 1). By construction (cf. Section II-A-2) any polyhedron explicitly consists of plane surfaces and, therefore, each cross section of the polyhedron within the $X Y$ plane at any position $z$ is given by a specific polygon. The polygon vertices change continuously along the $Z$ axis forming rays between the polyhedron vertices. The coordinates of all polygon vertices change linearly with the $z$ coordinate due to the theorem on intersecting lines. Now, the cross section of any polygon can be decomposed into elementary triangles whose sizes are defined by the appropriate vector products of the constituting triangle vectors, respectively. That is why the linear dependence of all polygon vertices on $z$ transforms to a dependence on $z$ of the polygon cross section that is quadratic at maximum. Therefore, the cross section in its most general form can be parametrized as second-order polynomial

$$
A(z)=C_{2} z^{2}+C_{1} z+C_{0} .
$$

The integration within the appropriate $z$ bounds provides the volume

$$
\begin{aligned}
V & =\int_{z_{1}}^{z_{2}} A(z) d z \\
& =\frac{1}{3} C_{2}\left(z_{2}^{3}-z_{1}^{3}\right)+\frac{1}{2} C_{1}\left(z_{2}^{2}-z_{1}^{2}\right)+C_{0}\left(z_{2}-z_{1}\right) .
\end{aligned}
$$

The three object dependent coefficients $C_{2}, C_{1}$, and $C_{0}$ are $a$ priori unknown, but can be derived by generating three constraints of the form

$$
A\left(\hat{z}_{\mu}\right)=C_{2} \hat{z}_{\mu}^{2}+C_{1} \hat{z}_{\mu}+C_{0} \equiv A_{\mu}
$$

with

$$
\hat{z}_{\mu} \in\left[z_{1}, z_{2}\right], \quad \mu=1,2,3 .
$$

${ }^{1}$ Analogous considerations are true for any other choice of the Cartesian coordinates. The orientation of the frame of reference can be arbitrarily chosen. 


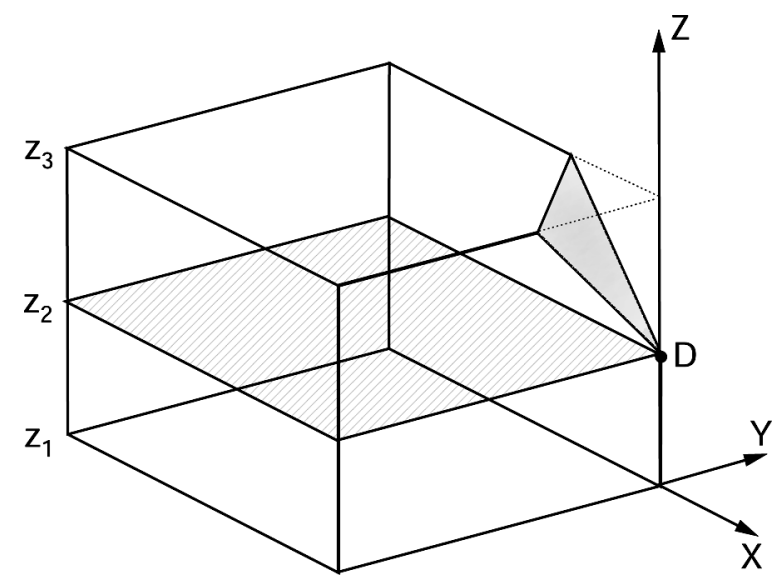

Fig. 2. Example of a valid polyhedron including a vertex of discontinuity (vertex D) at the coordinate $z=z_{2}$. Parametrization of the cross section according to (3) is separately valid for the intervals $\left[z_{1}, z_{2}\right]$ and $\left[z_{2}, z_{3}\right]$ with different values of $C_{i}$. Plane of segmentation (hatched) is defined as the $X Y$ plane including the vertex of discontinuity.

This means, that three cross sections at different coordinates $\hat{z}_{\mu}$ have to be evaluated (Fig. 1). Finding the values $A_{\mu}$ is then reduced to the evaluation of intersection polygons which are defined by the intersection between the polyhedron and planes parallel to the $X Y$ plane for three different sampling values $z=$ $\hat{z}_{\mu}$. Any value $A_{\mu}$ is determined by the size of the cross section of the corresponding sampled polygon. Thus, we obtain three linear (5) with respect to the requested coefficients $C_{i}$ which can be solved for $C_{i}$.

The above parametrization of vertex rays is valid for any polyhedron whose vertices are explicitly contained either within the $X Y$ plane with $z=z_{1}$ or within the $X Y$ plane with $z=$ $z_{2}$ (e.g., Fig. 1). Then, no additional polyhedron vertices exist in the parametrization interval $\left[z_{1}, z_{2}\right]$ and the applied method provides the exact volume without any approximation. However, for more complex polyhedrons, the method must be extended, because the direction of vertex rays along the $z$ axis and the corresponding parametrization change at any existing vertex (Fig. 2). Therefore, primarily a segmentation must be applied to separate discontinuities along the $z$ coordinate and ensure the validity of the above integration method. Sorting all vertices of the polyhedron along the $z$ coordinate generates $k$ intervals ${ }^{2}$ of the form $\left(\left[z_{1}, z_{2}\right],\left[z_{2}, z_{3}\right], \ldots,\left[z_{k}, z_{k+1}\right]\right)$ with $z_{\nu}<z_{\nu+1}$ and $\nu=1,2, \ldots, k$. Then, for each interval $\nu$ the calculation according to (4) can be performed separately without any discontinuities. Finally, the volume of all occurring segments must be added. It has to be emphasized that the applied method of volume calculation provides the exact result for any 3-D polyhedron with plane surfaces.

\section{B. Matrix Compression and Symmetry Conditioning}

Due to the sparseness of the system matrix, its computational representation can be efficiently reduced by sparse vectors where only nonzero elements and their corresponding voxel addresses (indices within an array) have to be stored. Nevertheless, typical matrix sizes quickly grow beyond the available

\footnotetext{
${ }^{2}$ The number of intervals $k$ only depends on the shape of the polyhedron and its orientation along the $z$ axis.
}

memory resources. Additional reduction can be achieved by exploiting internal symmetries where symmetric TORs with respect to the field of voxel have identical weights, but refer to appropriately mirrored voxels [1]. The voxels are typically stored in a one-dimensional (1-D) array where the numerical index addresses a specific voxel (Fig. 3, left). Consequently, the indices have to be transformed appropriately to get the correct addressing for symmetric TORs.

These time-intensive index transformations can completely be avoided when grouping voxels octant-wise, i.e., all voxels of a specific octant have to be stored in one 1-D array each. The new approach is illustrated in Fig. 3 for the 2-D case showing the modified enumeration patterns. All numerical indices are invariant with respect to any possible symmetry transformation. Only the global group addresses (assigned via a letter here) uniquely change, depending on the applied symmetry operation. Thus, the access to any sparse vector can nearly be performed as fast as for an uncompressed explicit representation and leads to significantly reduced reconstruction times.

The sketched concept can be extended to the 3-D case where the enumeration scheme is related to octants instead of quadrants, thus having additional groups. Our implementation handles four basic symmetries which are most common to PET systems, i.e., in the radial direction the $x$ symmetry, the $y$ symmetry or the $45^{\circ} x-y$ symmetry (Fig. 3, right) and in the axial direction the $z$ symmetry. ${ }^{3}$ Thus, the number of relevant matrix elements can be reduced by a factor of 16 at the cost of discriminating 24 different groups of voxels, i.e., three for each octant. Technically, the 24 groups of voxels are stored in one 1-D array each and sparse vectors store all addressed voxels group-wise. Then only the references of the addressed groups have to be modified via a lookup table $(24 \times 16$ pointers $)$ to transform a sparse vector to another symmetry configuration.

Typically, the symmetry handling would reduce the portability to other detector systems. However, our object-oriented implementation can easily be adapted to any detector system having the above mentioned four basic symmetries. Giving any set of (symmetric) sinograms, a sinogram sorter automatically detects symmetric TORs, matches them, and creates one associated sparse vector which effectively addresses up to $16 \mathrm{sym}$ metric TORs.

\section{Siemens/CTI ECAT HR+ Scanner}

The above methods have been applied for an iterative, fully 3-D reconstruction of 3-D sinograms recorded with a Siemens/CTI ECAT HR+ PET scanner consisting of 32 detector rings [20]. For the used default 3-D aquisition mode, the amount of data due to the large number of physical TORs is reduced by performing axial rebinning (span 9 , ring difference 22) and angular mashing (mash 2) with interleaving. Thus 239 sinograms with a radial pitch of $2.2 \mathrm{~mm}$ and an axial pitch of $2.42 \mathrm{~mm}$ are obtained. All presented images have been reconstructed from fully precorrected 3-D sinogram data [20] in 144 projections. In all cases, OSEM [11] with 12 subsets has been used. In total, approximately $4 \cdot 10^{6}$ TORs have been taken into account which correspond to a transaxial field of view of

\footnotetext{
${ }^{3}$ Other symmetries are not taken into account because this would restrict the voxel size to the geometry of the given sinograms.
} 


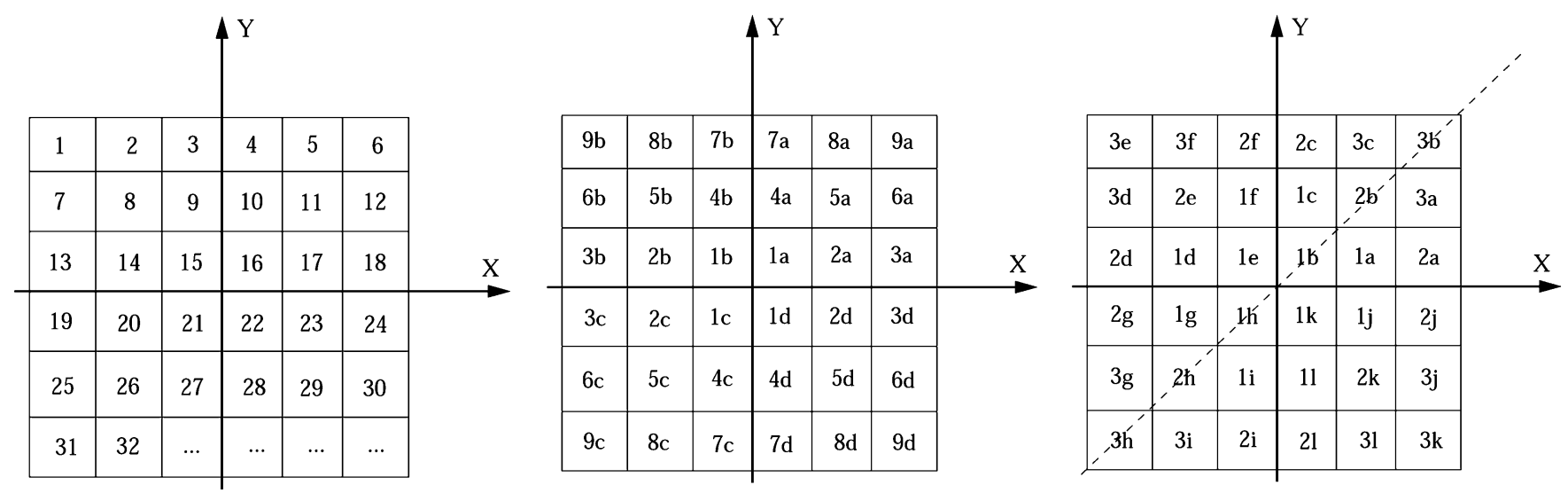

Fig. 3. Sketch of different enumeration schemes for the voxel addressing exemplarily for the 2-D case: simplest scheme (left), optimized access scheme for $x$ and $y$ symmetries only (middle) and optimized scheme for $x$ and $y$ symmetry and additional $45^{\circ} x-y$ symmetry (right); for the optimized scheme(s) only the characteristic group letter $(\mathrm{a}, \ldots, \mathrm{d})$ or $(\mathrm{a}, \ldots, \mathrm{k})$ has to be transformed corresponding to the applied symmetry operation via a simple lookup table.

$26 \mathrm{~cm}$ diameter appropriate for human brain measurements. In addition, the 3-D OSEM-one step late algorithm (OSMAPOSL) of the STIR library [21] in combination with its ray tracing projectors has been used for further comparisons.

\section{TOR Geometry}

For the used sinograms (Section II-C), any data bin is associated with a specific spatial TOR. The corresponding geometry must be generated to calculate the system matrix with VOI weighting according to Section II-A. First, the applied TOR dimensions are defined by the underlying radial and axial pitches of the sinograms. Second, the TOR position and orientation are uniquely determined by the radial and axial shifts with respect to the centre of field of view, the projection angle, and the specific inclination angle, depending on the addressed segment. Therefore, the TOR generation can be generalized by rotating and shifting each TOR polyhedron using these parameters.

\section{RESULTS}

\section{A. Evaluation of ${ }^{18}$ F-Measurements}

The applied continuous sampling of the image space using the VOI projectors results in a significant reduction of the level of noise compared to the LOI weighting. As proof, a homogenously ${ }^{18} \mathrm{~F}$-filled cylinder phantom has been measured and reconstructed either with VOI or LOI projectors for identical conditions, i.e., exactly the same voxelisation and input data. The level of noise in all reconstructed images has been quantified by calculating the square root of the variance relative to the mean value of the voxels within an identically positioned central region of interest.

Fig. 4 shows the level of noise as a function of the number of iterations for voxel sizes $2.2 \times 2.2 \times 2.42 \mathrm{~mm}^{3}$ and $1.4 \times 1.4 \times$ $2.42 \mathrm{~mm}^{3}$ of the reconstructed images. As expected, for any reconstruction, the level of noise increases with the number of iterations. For the two different voxel sizes, the curves obtained by the VOI reconstruction are nearly identical, whereas the LOI reconstruction always provides a higher level which even strongly degrades for the smaller voxels. The LOI weighting induces further sampling artefacts which significantly degrade the image

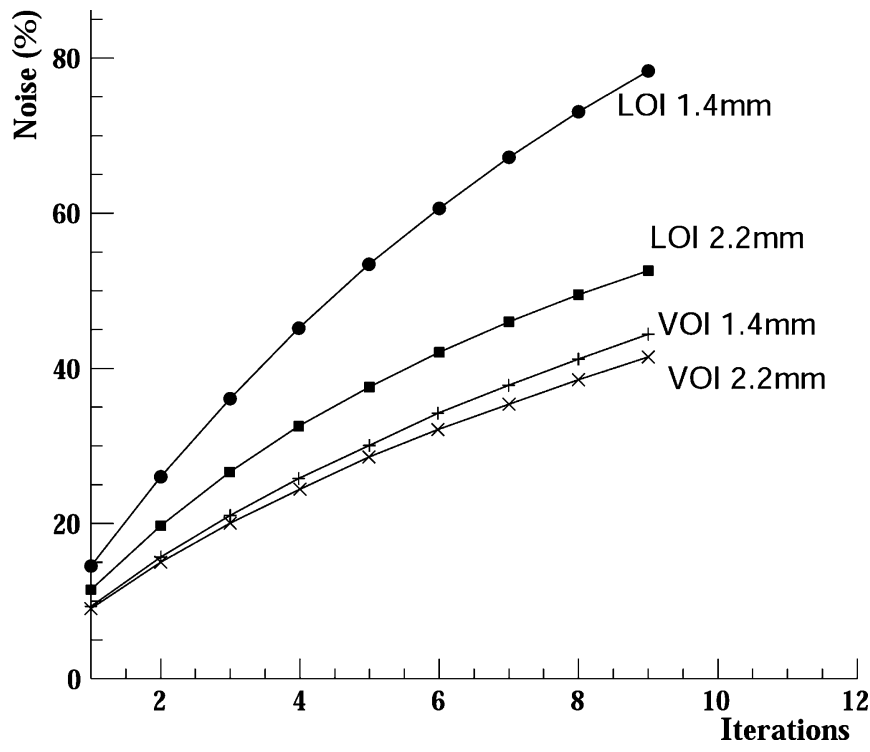

Fig. 4. Level of noise as a function of the number of iterations evaluated from reconstructed images (OSEM: 12 subsets) of a cylinder phantom with VOI and LOI weighting scheme for voxel sizes $2.2 \times 2.2 \times 2.42 \mathrm{~mm}^{3}$ and $1.4 \times 1.4 \times$ $2.42 \mathrm{~mm}^{3}$.

quality for voxel sizes smaller than the sampling pitches. Obviously, a uniformly continuous sampling of the image space is important to minimize the level of noise, especially when zooming has been applied.

Fig. 5 shows a comparison of the image quality for a 3-D hot rod Jaszczak phantom using either the VOI (left) or the LOI (right) reconstruction. The achieved noise reduction for the VOI sampling results in visibly better image quality. The smallest structures (4.8-mm rod diameters) which are close to the scanner resolution can only be identified for the lower level of noise.

In Fig. 6 a series of ${ }^{18}$ FDG human brain images (30 min scan) is presented. Again, the LOI weighting (right) provides images with a higher level of noise compared to the corresponding VOI weighting. Especially, for smaller voxels (voxel sizes $1.4 \times 1.4 \times 2.42 \mathrm{~mm}^{3}$; bottom), the different image quality becomes evident. For the VOI sampling, the granularity of the images is visibly reduced compared to the bigger voxel 


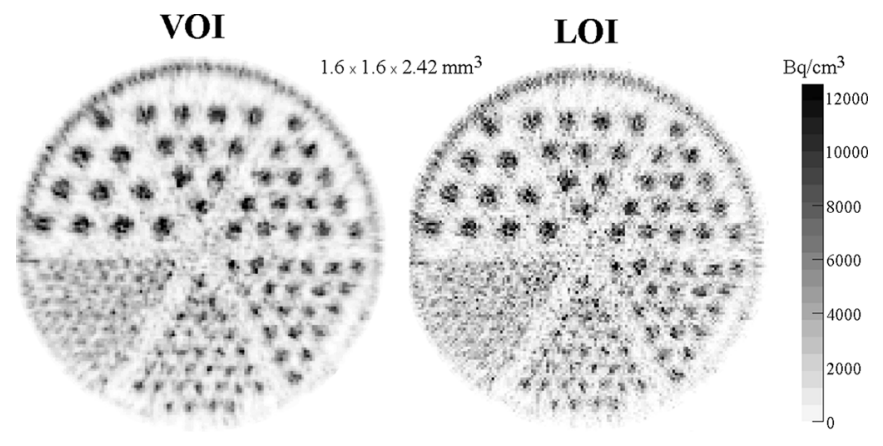

Fig. 5. Images of a ${ }^{18} \mathrm{~F}$-hot rod Jaszczak phantom (rod diameters 4.8, 6.4, 7.9, $9.5,11.1,12.7 \mathrm{~mm}$ ) reconstructed using VOI (left) and LOI (right) weighting.

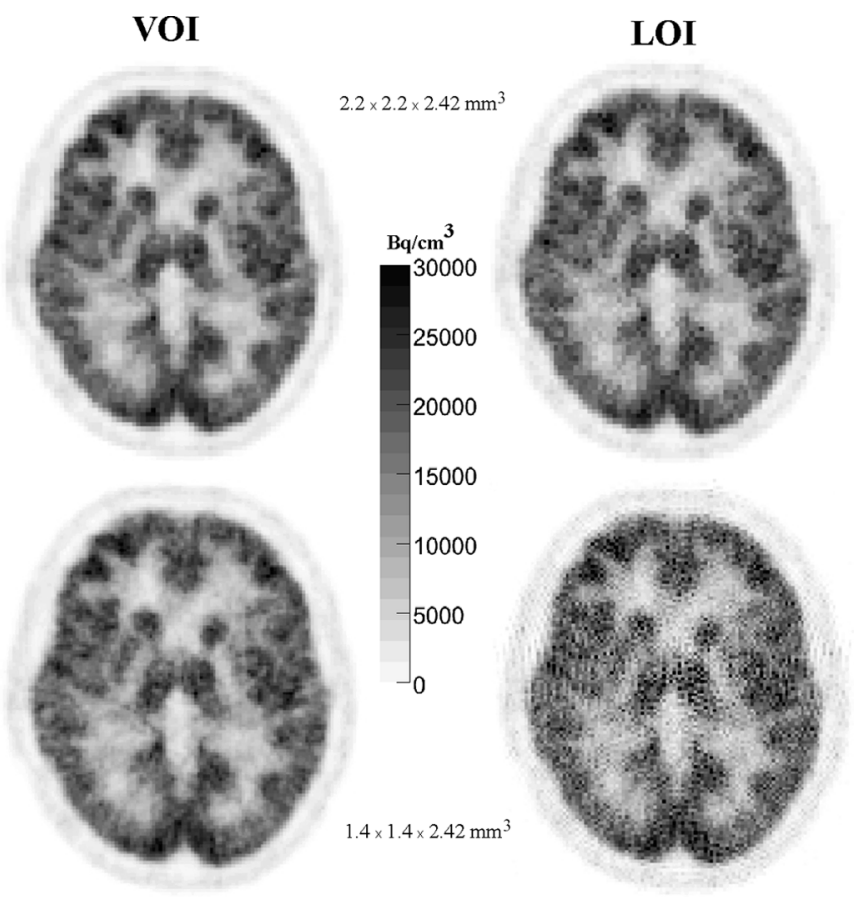

Fig. 6. Transaxial view of ${ }^{18} \mathrm{FDG}$ human brain images reconstructed using VOI weighting (left) and LOI weighting (right); different voxelisations have been used: $2.2 \times 2.2 \times 2.42 \mathrm{~mm}^{3}$ (top) and $1.4 \times 1.4 \times 2.42 \mathrm{~mm}^{3}$ (bottom).

size. Consequently, object edges are smoothed without significant enhancement of noise. However, for the LOI sampling, a zooming provides strongly degraded images (bottom, right). The level of noise grows intolerably and even ring artefacts can be detected because of the coarse sampling.

Besides a zooming within the transaxial planes, the VOI reconstruction also allows a regular axial zooming. Due to the continuous sampling of the image space, no geometrical fit between TORs and the voxel dimensions and accordingly positioning in all three dimensions is needed. As described in Section II-A-2, any TOR is splitted according to the given planes of all touched voxels, independent of the applied voxel configuration. In this way, the VOI approach is absolutely robust against sampling artefacts and makes a simple handling of the projector possible for any geometrical situation. This is shown in Fig. 7. The granularity of the image can be reduced in all three dimensions without introducing any visible artefacts and the accompanied unavoidable enhancement of the level of noise remains

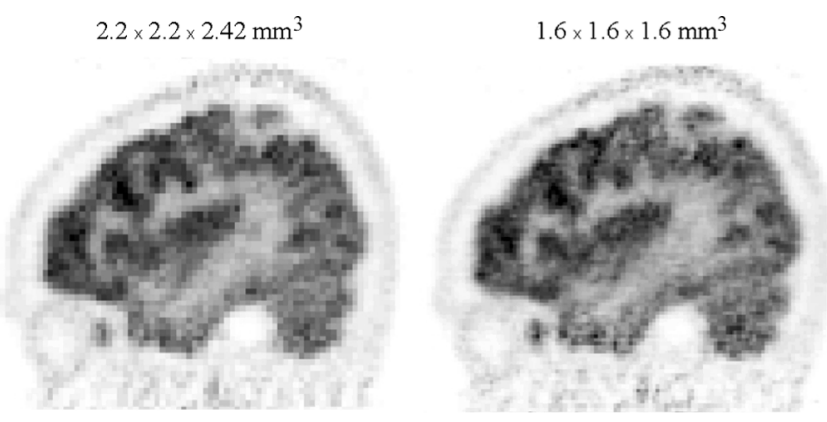

Fig. 7. Axial (sagital) view of a ${ }^{18} \mathrm{FDG}$ human brain image reconstructed using VOI weighting without (left) and with (right) axial+transaxial zooming for the same display range as Fig. 6.

acceptable. Here, the LOI weighting provides only corrupted reconstruction results.

The dependence between contrast and noise for different reconstructions is shown in Fig. 8. A ${ }^{18} \mathrm{~F}$-filled hot sphere has been measured with a true ratio of 3.15 with respect to the background environment. The sphere with a diameter of $26.2 \mathrm{~mm}$ has been evaluated by defining a centered ROI with a diameter of 18 $\mathrm{mm}$. The left plot gives the curves resulting from VOI projector and LOI projector for two different voxel sizes of $2.2 \times 2.2 \times$ $2.42 \mathrm{~mm}^{3}$ and $1.4 \times 1.4 \times 2.42 \mathrm{~mm}^{3}$. The contrast-noise curves are nearly independent of the voxel size for the VOI projector whereas the LOI projector shows a significant dependence. The VOI projector gives a better contrast-noise level in any case. In addition, a comparison with the 3-D OSMAPOSL reconstruction of STIR [21] using the ray tracing technique is shown (right plot). The differences of the contrast for identical levels of noise amount to $8 \%-10 \%$ in which the VOI projector systematically provides the higher contrast value without pronounced dependence upon the voxel size.

\section{B. Technical Performance}

The utilization of the above methods (Section II-B) allows a very fast, iterative reconstruction as requested for routine applications. At first, consideration of four symmetries gives a reduction factor of 16 for the weights to be stored. Then, the proposed voxel addressing enables the access to all matrix weights of the compressed matrix nearly as fast as to those of its uncompressed analogon. The symmetry transformations need less than one percent of the total reconstruction time.

Finally, due to the full memory-resident storage the reconstruction time is achieved independent of any effort to calculate complex, more accurate matrix weights, i.e., the calculation time for any projector model (VOI/LOI) does not influence the reconstruction time.

Exemplarily, properties of system matrix for voxel sizes $2.2 \times$ $2.2 \times 2.42 \mathrm{~mm}^{3}$ (cf. Fig. 6, top views) are presented in Table I for VOI and LOI weighting. For the VOI matrix calculations, the number of matrix elements is significantly larger compared to the simple LOI calculations, since much more voxels have been touched for spatially extended TORs. The matrix size in terms of allocated memory changes equivalently, but the maximum size remains manageable with standard hardware. All reconstructions have been performed on a Linux PC with $3.0-\mathrm{GHz}$ Intel 

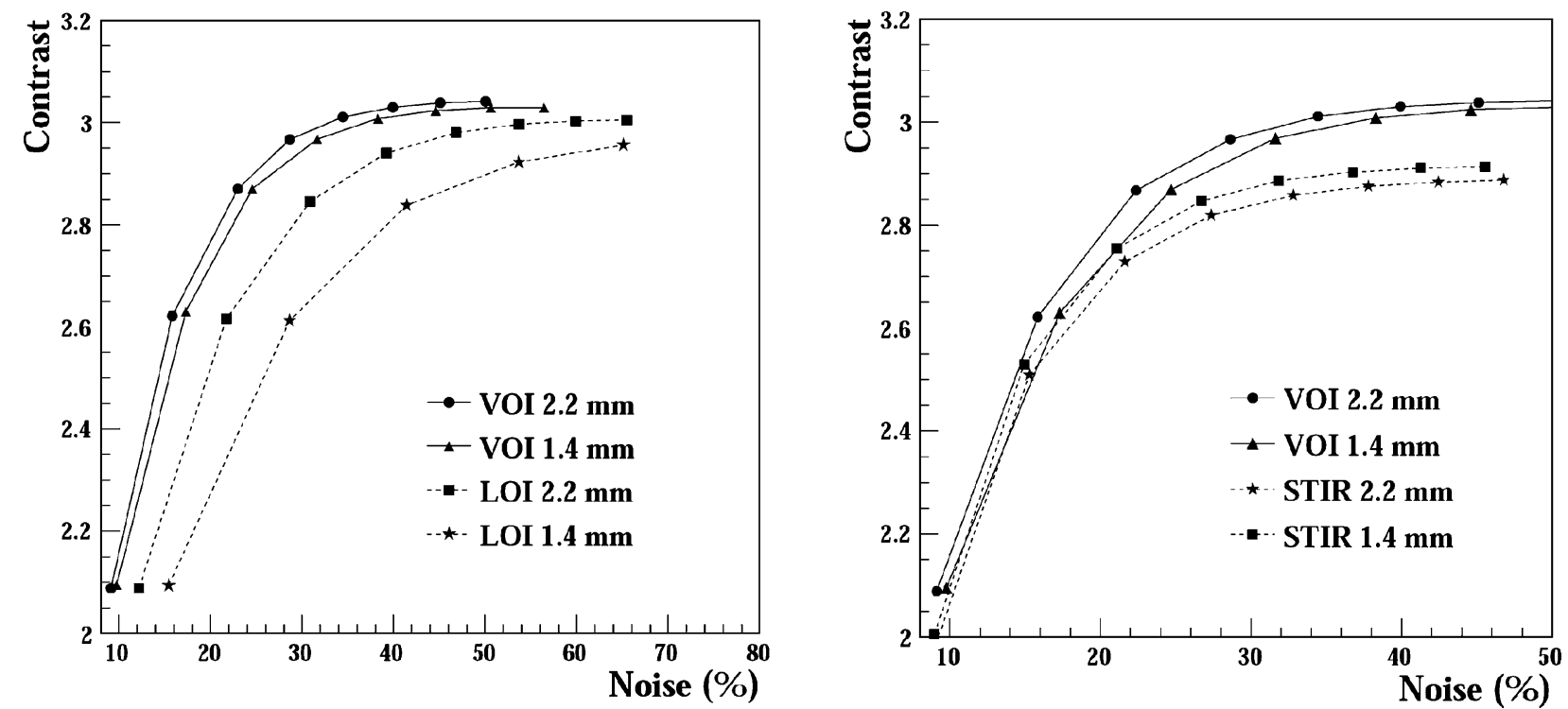

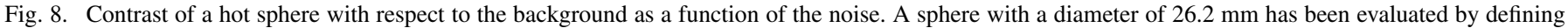

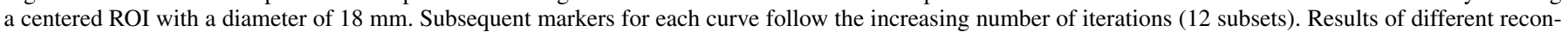

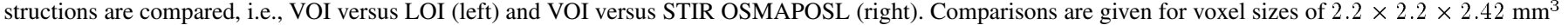
and $1.4 \times 1.4 \times 2.42 \mathrm{~mm}^{3}$.

TABLE I

COMPARISON OF THE BASIC PROPERTIES OF THE COMPRESSED SYSTEM MATRIX USING THE VOI OR LOI PROJECTOR

\begin{tabular}{|l||c|c|}
\hline \multicolumn{1}{|l||}{ Dimensions } & \multicolumn{2}{|c|}{$116 \times 116 \times 64$} \\
\hline Voxel Size & $2.2 \times 2.2 \times 2.42 \mathrm{~mm}^{3}$ \\
\hline Number of TORs & \multicolumn{2}{|c|}{$\approx 4 \cdot 10^{6}$} \\
\hline \hline Weighting & VOI & LOI \\
\hline Number of Stored Weights & $95 \cdot 10^{6}$ & $30 \cdot 10^{6}$ \\
\hline Matrix Size (compressed) & $0.9 \mathrm{~GB}$ & $0.4 \mathrm{~GB}$ \\
\hline Matrix Pre-Calculation time & $110 \mathrm{~min}$ & $3.5 \mathrm{~min}$ \\
\hline Recon. Time (1 of 12 subsets) & $7.6 \mathrm{~s}$ & $3.4 \mathrm{~s}$ \\
\hline
\end{tabular}

Pentium processor and 2-GB memory. The complete matrix calculations for the setup in Table I using VOI weighting needs less than $2 \mathrm{~h}$ CPU time without any parallelisation. Restoring the matrix from a local disc only takes a few seconds. The effective reconstruction time is dominated by the number of floating point operations which is approximately proportional to the amount of matrix weights. For the applied setup with a compressed matrix of nearly $1 \mathrm{~GB}$ size and using OSEM with 12 subsets, one subset iteration is executed in $7.6 \mathrm{~s}$ CPU time. Therefore, complex synchronised parallelisation schemes and specific hardware are not needed for our iterative, fully 3-D reconstruction with VOI projectors.

\section{Discussion}

A memory-resident, precalculable system matrix [1] in conjunction with the proposed new symmetry handling allows a very fast, iterative, fully 3 -D reconstruction. Thus, the time of reconstruction is completely independent of the needed time to evaluate any kind of projectors. This approach has been exploited to implement an analytical VOI projector which has not been applied in PET before. In contrast to a simple LOI weighting [5], the VOI weighting provides a uniformly continuous sampling of the image space. Due to improved regularization of the system matrix, the level of noise can be significantly reduced and the reconstruction remains stable in case of zooming, i.e., for voxel sizes much smaller than the effective data sampling suggests. The stability and robustness of the VOI projector makes any fine-tuning between voxel dimensioning and data sampling unessential to obtain undistorted images. Therefore, the VOI projector becomes easily adaptable to any geometrical situation and allows a flexible and fully user-defined setting of voxel sizes in the transaxial and axial dimensions. The approach can be adapted to any kind of iterative reconstructions [9]-[19] without restrictions. Also, a combination with dedicated blurring models [22]-[24] is feasible.

Instead of considering only one single LOR for each TOR, a whole bunch of parallel LORs could be evaluated to approximately mimic the VOI weighting. However, the achieved accuracy is related to the number of internally sampled LORs and the effort increases necessarily for higher precision. Our analytical method directly provides the correct weights without limited accuracy.

Compared to the VOI projector a more appropriate projector which considers the spatially variant covered solid angle of the detector crystals [22] would also be feasible with the precalculable system matrix. But here a major merit is only expected when evaluating all true TORs spanned from any detected crystal combination, i.e., having a full LM reconstruction [2], [3] appropriately considering the crystal sizes and positioning. However, the VOI projector would also benefit from such LM reconstruction. In contrast, standard sinograms using geometrical constructs of direct/indirect slices and interleaving with pitches half of the true crystal pitches [20], but particularly with rebinned/reduced data introduce binning losses. Then the potential of a more dedicated modeling of solid angle dependencies could partially be foiled. In fact, the VOI weighting depicts a good approximation of the detection probabilities for 3-D sinogram-based data which is superior to discontinuous sampling methods with respect to flexibility and accuracy. 


\section{ACKNOWLEDGMENT}

The authors would like to thank Y. Li, D. Krücker, and E. Rota Kops for valuable discussions, as well as L. Tellmann for technical support.

\section{REFERENCES}

[1] C. A. Johnson, Y. Yan, R. E. Carson, R. L. Martino, and M. E. DaubeWitherspoon, "A system for the 3-D reconstruction of retracted-septa PET data using the EM algorithm," IEEE Trans. Med. Imag., vol. 42, no. 4, pp. 1223-1227, Aug. 1995.

[2] T. E. Nichols, J. Qi, E. Asma, and R. M. Leahy, "Spatiotemporal reconstruction of list-mode PET data," IEEE Trans. Med. Imag., vol. 21, no. 4, pp. 396-404, Apr. 2002.

[3] R. H. Huesmann, G. J. Klein, W. W. Moses, J. Qi, B. W. Reutter, and P. Virador, "List-mode maximum-likelihood reconstruction applied to Positron Emission Mammography (PEM) with irregular sampling," IEEE Trans. Med. Imag., vol. 19, no. 5, pp. 532-537, May 2000.

[4] X. Liu, M. Defrise, C. Michel, M. Sibomana, C. Comtat, P. Kinahan, and D. Townsend, "Exact rebinning methods for three-dimensional PET," IEEE Trans. Med. Imag., vol. 18, no. 8, pp. 657-664, May 1999.

[5] R. L. Siddon, "Fast calculation of the exact radiological path for threedimensional CT array," Med. Phys., vol. 12, pp. 252-255, Mar. 1985.

[6] K. Ziemons, H. Herzog, and L. E. Feinendegen, "Iterative image reconstruction with weighted pixel contributions to projection elements," Eur. J. Nucl. Med., vol. 16, p. 403, 1990.

[7] A. Terstegge, S. Weber, H. Herzog, H. W. Mueller-Gartner, and H. Hailing, "High resolution and better quantification by tube of response modelling in 3-D PET reconstruction," in Proc. IEEE Nucl. Sci. Symp. Med. Imag. Conf., Anaheim, CA, 1996, pp. 1603-1607.

[8] J. J. Scheins, Tomographic reconstruction of transverse and longitudinal phase space distributions using the maximum entropy algorithm TESLA Collaboration, DESY, Hamburg, Germany, TESLA Rep. 2004-08, May 2004.

[9] L. Shepp and Y. Vardi, "Maximum likelihood reconstruction for emission tomography," IEEE Trans. Med. Imag., vol. 1, pp. 113-122, 1982.

[10] K. Lange and R. Carson, "EM reconstructionn algorithms for emission and transmission tomography," J. Comput. Assist. Tomogr., vol. 8, pp. 306-316, 1984.

[11] H. Hudson and R. Larkin, "Accelerated image reconstruction using ordered subsets of projection data," IEEE Trans. Med. Imag., vol. 13, no. 4, pp. 601-609, Dec. 1994.
[12] T. J. Hebert and R. Leahy, "Fast methods for including attenuation in the EM algorithm," IEEE Trans. Nucl. Sci., vol. 13, no. 2, pp. 89-101, Apr. 1994.

[13] J. Qi, M. Leahy, C. Hsu, T. H. Farquhar, and S. R. Cherry, "Fully 3-D Bayesian reconstruction for the ECAT EXACT HR+," in Proc. IEEE Nucl. Sci. Med. Imag. Symp., 1997, vol. 2, pp. 1042-1046.

[14] M. Yavuz and J. Fessler, "New statistical models for randoms-precorrected PET scans," in Proc. IPMI, 1997, pp. 190-203.

[15] X. Liu, C. Comtat, C. Michel, P. Kinahan, M. Defrise, and D. Townsend, "Comparison of 3-D reconstruction with 3-D-OSEM and with FORE+OSEM for PET," IEEE Trans. Med. Imag., vol. 20, no. 8, pp. 804-814, Aug. 2001.

[16] C. Michel, M. Sibomana, A. Bol, X. Bernard, M. Lonneux, M. Defrise, C. Comtat, P. E. Kinahan, and D. W. Townsend, "Preserving poisson characteristics of PET data with weighted OSEM reconstruction," in Proc. IEEE Nucl. Sci. Symp. Med. Imag. Conf., 1998, vol. 2, pp. $1323-1329$.

[17] C. Comtat, P. E. Kinahan, M. Defrise, C. Michel, and D. W. Townsend, "Fast reconstruction of 3-D PET data with accurate statistical modeling," IEEE Trans. Nucl. Sci., vol. 45, no. 3, pp. 1083-1089, Jun. 1998.

[18] S. Alenius and U. Ruotsalainen, "Bayesian image reconstruction for emission tomography based on median root prior," Eur. J. Nucl. Med., vol. 24, pp. 258-265, 1997.

[19] E. Mumcuoglu, R. Leahy, S. R. Cherry, and Z. Zhou, "Fast gradientbased methods for Bayesian reconstruction of transmission and emission PET images," IEEE Trans. Med. Imag., vol. 13, no. 4, pp. 687-701, Dec. 1994.

[20] ECAT EXACT HR+ Operating Instructions, Siemens Medical Systems Inc., Knoxville, TN, 1996.

[21] C. Labbe, K. Thielemans, H. Zaidi, and C. Morel, "An object-oriented library incorporating efficient projection/backprojection operators for volume reconstruction in 3-D PET," presented at the Proc. 3D99 Conf., Egmond aan Zee, The Netherlands, 1999.

[22] J. Qi, R. M. Leahya, S. R. Cherry, A. Chatziioannou, and T. H. Farquhar, "High-resolution 3-D Bayesian image reconstruction using the microPET small-animal scanner," Phys. Med. Biol., vol. 43, pp. 1001-1013, 1998.

[23] A. J. Reader, P. J. Julyan, H. Williams, D. L. Hasting, and J. Zweit, "EM algorithm system modeling by image-space convolution technique for PET reconstruction," IEEE Trans. Nucl. Sci., vol. 50, no. 5, pp. 1392-1397, Oct. 2003.

[24] J. Doll, M. Henze, O. Bublitz, A. Werling, L. E. Adam, U. Haberkorn, W. Semmler, and G. Brix, "High Resolution reconstruction of PET images using the iterative OSEM algorithm," Nuklearmedizin, vol. 43, pp. 72-78, 2004. 